J. Indones. Math. Soc.

Special Edition (2011), pp. 1-9.

\title{
GRACEFUL LABELING ALGORITHMS AND COMPLEXITY - A SURVEY
}

\author{
S. Arumugam ${ }^{1,2}$, Jay Bagga $^{3}$ \\ ${ }^{1}$ National Centre for Advanced Research in Discrete Mathematics \\ (n-CARDMATH), Kalasalingam University, Anand Nagar, \\ Krishnankoil-626190, India, s.arumugam.klu@gmail.com \\ ${ }^{2}$ School of Electrical Engineering and Computer Science, \\ The University of Newcastle, NSW 2308, Australia. \\ ${ }^{3}$ Department of Computer Science, Ball State University, \\ Muncie, IN 47306, USA, jbagga@bsu.edu
}

\begin{abstract}
Graceful graphs were first studied by Rosa in 1966. The Kotzig-Ringel graceful tree conjecture states that every tree has a graceful labeling. Aldred and McKay and others have used computer programs to show that trees of order up to 35 are graceful. Bagga et al. investigated algorithms for generating all graceful labelings of certain known classes of graceful graphs, including paths, cycles, and certain other classes of unicyclic graphs. The data generated by such algorithms has led to the discovery of new properties of such graceful labelings. In this paper we present a survey of graceful graph labeling algorithms and related complexity issues.

Key words: Graph labelings, graceful labeling, algorithms, complexity.
\end{abstract}

2000 Mathematics Subject Classification: 05C30, 05C78, 05C85. Received: 09-08-2011, revised: 09-09-2011, accepted: 04-12-2012 